\title{
Resource Allocation via Linear Programming for Multi-Source, Multi-Relay Wireless Networks
}

\author{
Nariman Farsad and Andrew W. Eckford \\ Dept. of Computer Science and Engineering, York University \\ 4700 Keele Street, Toronto, Ontario, Canada M3J 1P3 \\ E-mail: nariman@cse.yorku.ca, aeckford@yorku.ca
}

\begin{abstract}
In a cooperative wireless network, there may be many potential relays within radio range of a source; similarly, there may be many potential sources seeking to use relays. Allocating these resources is a non-trivial optimization problem. In this paper, fractional cooperation is considered, where each potential relay only allocates a fraction of its resources to relaying. It is shown that linear programming can be used to optimally allocate resources in multi-source, multi-relay networks, where the relays use a demodulate-and-forward (DemF) strategy, and where the transmissions are protected by lowdensity parity-check (LDPC) codes. Compared with existing optimization schemes, this method is particularly suitable for very large networks with numerous sources and relays. Simulation results are presented to demonstrate the performance of this scheme.
\end{abstract}

\section{INTRODUCTION}

In wireless networks, spatial distribution of nodes generally results in independent fading on different links. This spatial distribution can be exploited in cooperative diversity [1], [2], where each node can assist its neighbours in transmitting information to a data sink. In its simplest form, a cooperative system is a relay system consisting of three nodes: a source, a relay, and a destination. The relay can use various cooperative schemes such as decode-and-forward (DF) [3] and demodulate-and-forward (DemF) [4], [5], to assist the source in transmitting its information bits to the destination. DemF is particularly applicable to devices with reduced computational abilities, such as in wireless sensor networks (WSNs).

In most wireless networks, a source node is typically in radio range of multiple relays. Eckford et al. [6] introduced fractional cooperation as a low-complexity cooperative scheme for such multi-relay systems, to be used in conjunction with DemF. Using this scheme, a large number of relays retransmit a small fraction of the source's transmission bits, so that the relaying cost is spread over a large number of relays instead of one - thus, each relay contributes "as much as it can," while reserving resources for the transmission of its own information.

A key challenge in fractional cooperation is resource allocation, in which the system determines what fraction must be selected for retransmission by each relay. This challenge is exacerbated in systems with multiple sources, all of which are competing for the same fractional resource at the relays. In

This work was financially supported by a Discovery Grant from the Natural Sciences and Engineering Research Council. this paper, we pose optimal resource allocation in fractional cooperation as a convex optimization problem, ensuring successful transmission while minimizing energy consumption. In particular, we use linear programming to minimize the number of transmission bits subject to the constraint that decoding at the destination is successful. This constraint is derived using extrinsic information transfer (EXIT) charts [7].

Our approach is partly inspired by [8], in which linear programming was used in combination with EXIT charts in order to optimize LDPC degree sequences. Further codeoptimization work for relay channels was given in [9]-[11], though the current paper concerns resource allocation rather than code optimization. Related work on resource allocation was done in [12]. However, in that paper, the union bound was used for analysis (rather than EXIT charts), which led to a nonconvex optimization problem. The current paper is a significant improvement on that work: by stating the optimization problem in terms of linear programming, one is guaranteed to efficiently find a global optimum, regardless of the number of sources and relays. Furthermore, we give simulation results showing the performance of our approach, which is especially suitable for large (many-source, manyrelay) networks. Although we focus on DemF cooperation, our methods can also be applied to DF, which we will discuss in future work.

The rest of this paper is organized as follows. In section II, we present a multi-source, multi-relay system model that represents a wireless network employing fractional cooperation. In section III, we formulate a linear programming model that will ensure successful transmission while optimizing the transmission power. In section IV, we verify the validity of our linear programming model by presenting simulation results.

\section{System MOdeL}

\section{A. Relay model}

The relay model that we use is an extension of the fractional cooperation model proposed in [6]: we consider $s$ sources, $r$ relays, and a single destination. The $r$ relays are shared amongst all $s$ sources (i.e. each single source has $r$ relays that assists in its transmission), as shown in Figure 1. Each source measures a phenomenon, encodes it using LDPC codes, and broadcasts the encoded codeword to the $r$ relays, as well as the destination. Using DemF, the $j$ th relay makes hard decisions, selects a small fraction $\epsilon_{j}$ (where, in general, $\epsilon_{i} \neq \epsilon_{j}$ for 


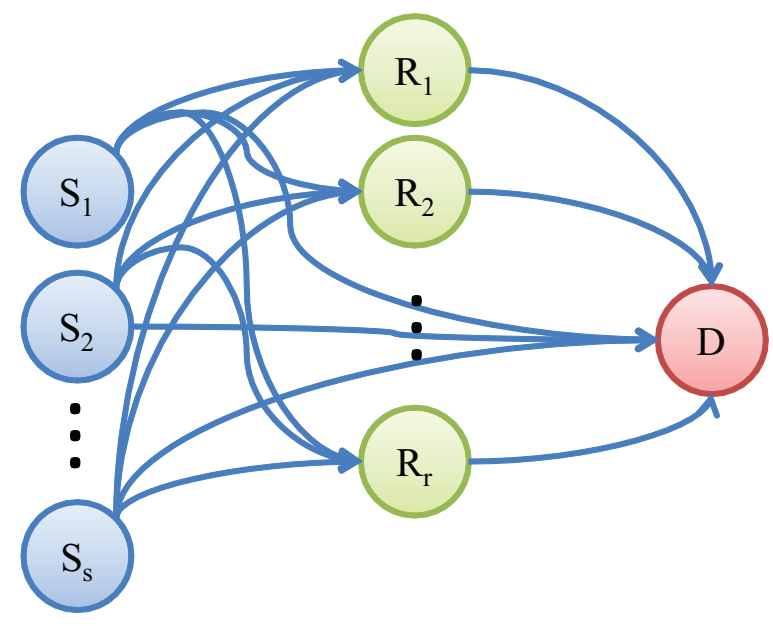

Fig. 1. Multi-Source, Multi-relay model.

$i \neq j$ ), re-encodes them using LDPC codes, and transmits the resulting codeword to the destination. The destination will then decode each source's information bits using the received signal from the $r$ relays, as well as the source itself.

Each source has a length- $n$ information sequence to transfer to the destination represented by $\mathbf{x}^{\left(S_{i}\right)}=$ $\left[x_{1}^{\left(S_{i}\right)}, x_{2}^{\left(S_{i}\right)}, \ldots, x_{n}^{\left(S_{i}\right)}\right]$, where $x_{k}^{\left(S_{i}\right)} \in\{0,1\}$ and $S_{i}$ represents the $i$ th source. Each information sequence is encoded by an LDPC code for each source. Let $R_{1}^{\prime}, R_{2}^{\prime}, \cdots, R_{s}^{\prime}$ be the code rates at each source. Therefore the codeword ready for transmission at the $i$ th source is represented by $\mathbf{z}^{\left(S_{i}\right)}=$ $\left[z_{1}^{\left(S_{i}\right)}, z_{2}^{\left(S_{i}\right)}, \ldots, z_{c_{i}}^{\left(S_{i}\right)}\right]$, where $c_{i}=n / R_{i}^{\prime}$ is the length of the codeword.

As shown in Figure 1, there are $s r$ source to relay (S-R), $s$ source to destination (S-D), and $r$ relay to destination (RD) links. We assume these communication links use binary phase shift keying (BPSK) for data modulation. We define the function $\phi:\{0,1\} \rightarrow\{+1,-1\}$ as the modulation function where 0 is mapped to $a+1$ and 1 is mapped to -1 . With slight abuse of the inverse notation, the demodulation function is defined as

$$
\phi^{-1}(y)=\left\{\begin{array}{ll}
0 & \text { if } y \geq 0 \\
1 & \text { otherwise }
\end{array},\right.
$$

The S-D links are therefore given by

$$
\mathbf{y}^{\left(S_{i}, D\right)}=\phi\left(\mathbf{z}^{\left(S_{i}\right)}\right)+\mathbf{n}^{\left(S_{i}, D\right)},
$$

where $S_{i}$ corresponds to the $i$ th source and $\mathbf{n}^{\left(S_{i}, D\right)}$ is AWGN with variance $\sigma_{\left(S_{i}, D\right)}^{2}$. The channel SNRs for each of the $s$ S-D links are represented by $\gamma_{\left(S_{i}, D\right)}=1 /\left(2 \sigma_{\left(S_{i}, D\right)}^{2}\right)$.

The S-R links are also given by

$$
\mathbf{y}^{\left(S_{i}, R_{j}\right)}=\phi\left(\mathbf{z}^{\left(S_{i}\right)}\right)+\mathbf{n}^{\left(S_{i}, R_{j}\right)},
$$

where $S_{i}$ and $R_{j}$ correspond to the $i$ th source and the $j$ th relay respectively and $\mathbf{n}^{\left(S_{i}, R_{j}\right)}$ is AWGN with variance $\sigma_{\left(S_{i}, R_{j}\right)}^{2}$. Therefore, all the S-R links can be represented by $s r$ channel SNRs, $\gamma_{\left(S_{i}, R_{j}\right)}=1 /\left(2 \sigma_{\left(S_{i}, R_{j}\right)}^{2}\right)$.

\section{B. Demodulate-and-forward}

In DemF, a relay first demodulates the signal received from a source. This process can be formulated as

$$
\mathbf{z}^{\left(S_{i}, R_{j}\right)}=\phi^{-1}\left(\mathbf{y}^{\left(S_{i}, R_{j}\right)}\right),
$$

where $\mathbf{z}^{\left(S_{i}, R_{j}\right)}$ is the results of hard decisions (demodulation) for the $j$ th relay assisting $i$ th source. Each relay then selects a fraction of the demodulated signal, re-encodes it using error correcting codes and retransmits to the destination.

The vector $\mathbf{b}^{\left(S_{i}, R_{j}\right)}$ represents the demodulated bit positions selected for retransmission to the destination: if $b_{k}^{\left(S_{i}, R_{j}\right)}=1$, then the $k$ th bit is relayed; if $b_{k}^{\left(S_{i}, R_{j}\right)}=0$, then the $k$ th bit is not relayed. Therefore, the demodulated sequence resulting from the $j$ th relay assisting $i$ th source is available at the destination as

$$
\mathbf{y}_{D e m F}^{\left(S_{i}, R_{j}, D\right)}=\mathbf{b}^{\left(S_{i}, R_{j}\right)} \odot \phi\left(\mathbf{z}^{\left(S_{i}, R_{j}\right)}\right),
$$

where $\odot$ is element-wise multiplication of vectors, $\mathbf{z}^{\left(S_{i}, R_{j}\right)}$ is given by equation (4), and $\mathbf{y}_{D e m F}^{\left(S_{i}, R_{j}, D\right)}$ represents the results of demodulations available at the destination. The elements of $\mathbf{y}_{\text {DemF }}^{\left(S_{i}, R_{j}, D\right)}$ can take three possible values: +1 (representing a demodulated 0 bit), -1 (representing a demodulated 1 bit), and 0 (representing a position that is not selected for relaying).

The channel LLR for the S-D link is calculated as

$$
\boldsymbol{\ell}^{\left(S_{i}, D\right)}=2 \mathbf{y}^{\left(S_{i}, D\right)} / \sigma_{\left(S_{i}, D\right)}^{2}=4 \gamma_{\left(S_{i}, D\right)} \mathbf{y}^{\left(S_{i}, D\right)},
$$

and for the S-Rs link as

$$
\boldsymbol{\ell}_{D e m F}^{\left(S_{i}, R_{j}, D\right)}=\mathbf{y}_{D e m F}^{\left(S_{i}, R_{j}, D\right)} \log \left[\frac{1-p_{D e m}^{\left(S_{i}, R_{j}\right)}}{p_{D e m}^{\left(S_{i}, R_{j}\right)}}\right],
$$

where $p_{\text {Dem }}^{\left(S_{i}, R_{j}\right)}$ is the probability of demodulation error at the $j$ th relay assisting $i$ th source given by

$$
p_{D e m}^{\left(S_{i}, R_{j}\right)}=\frac{1}{2} \operatorname{erfc}\left(\sqrt{\gamma_{\left(S_{i}, R_{j}\right)}}\right) .
$$

Consequently, the message LLR input to the iterative LDPC decoder of the $i$ th source can be calculated as

$$
\boldsymbol{\ell}_{D e m F}^{\left(S_{i}\right)}=\boldsymbol{\ell}^{\left(S_{i}, D\right)}+\sum_{j=1}^{r} \ell_{D e m F}^{\left(S_{i}, R_{j}, D\right)}
$$

In DemF any type of code (such as RA codes or irregular LDPC codes) can be used. For simplicity we assume that using powerful and capacity approaching codes such as RA [14], and irregular LDPC [15] codes, over R-D links, can result in perfect recovery of demodulated bits at the destination, at rates close to capacity. Thus, for any DemF system, we will assume that a capacity-approaching code is used in the RD link, and is decoded without error. However, for a fixed number of information bits, the total number of transmitted bits over the R-D link will depend on the capacity, which is a function of the link SNR, written $\gamma_{R_{i}, D}$ for the $i$ th relay. 


\section{Linear Programming Model}

In this section we present a linear programming model that will minimize the number of transmission bits of a multisource multi-relay system, described in the previous section, subject to the constraint of successful transmission. In the next section we will confirm these methods by presenting simulations.

\section{A. Key Assumptions and Definitions}

We consider the multi-relay, multi-source system, explained in section II, with $r$ relays and $s$ sources. For each source, we have $r$ relays, and therefore $r$ source-relay (S-R) and relaydestination (R-D) links (as well as a single source-destination (S-D) link). Without loss of generality, we assume that the all-zero codeword is transmitted by each source, which is equivalent to the all- $(+1)$ channel codeword. We assume all links are independent AWGN channels, represented with their respective channel SNR.

Define a vector of length $s, \gamma_{S D}$, as

$$
\gamma_{S D}=\left[\begin{array}{llll}
\gamma_{\left(S_{1}, D\right)} & \gamma_{\left(S_{2}, D\right)} & \cdots & \gamma_{\left(S_{s}, D\right)}
\end{array}\right]^{T} .
$$

As explained in section II, for simplicity, we assume that for DemF the R-D links are perfect. We define $\epsilon_{\left(S_{i}, R_{j}\right)}$ as the fraction of $i$ th source transmission that was forwarded by the $j$ th relay. We define a vector of length $s r, \boldsymbol{\epsilon}$, as

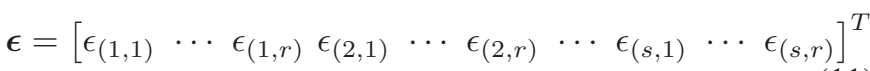

where the elements represent the fractions that are selected for retransmission by each relay for each source.

In [8] it was shown that messages passed in the LDPC decoder can be (approximately) represented using a single parameter - in that paper, message error probability was used, but in this paper, we use the mean of the LLR messages. Therefore, for LDPC codes, an approximate minimum channel LLR mean required for successful decoding, written $m_{\ell_{\text {min }}}$, can be calculated using simulations and EXIT chart analysis. Hence, we can assume that if the channel LLR mean, $m_{\ell}$, that is input to the iterative decoding algorithm, satisfies $m_{\ell} \geq m_{\ell_{\text {min }}}$, the iterative decoding process is assumed to be successful.

Since for each source we can have a different minimum channel LLR mean requirements, we define a vector of length $s, \mathbf{m}_{\ell_{\text {min }}}$, as vector with $s$ elements that are

$$
\mathbf{m}_{\ell_{\text {min }}}=\left[\begin{array}{llll}
m_{\ell_{m i n}}^{\{1\}} & m_{\ell_{m i n}}^{\{2\}} & \cdots & m_{\ell_{m i n}}^{\{s\}}
\end{array}\right]^{T},
$$

where $m_{\ell_{\text {min }}}^{\{i\}}$ is the minimum channel LLR mean threshold for the $i$ th source.

\section{B. Successful Transmission Requirements}

As described in the previous section, in order to ensure successful transmission and decoding at the destination, the mean of the input channel LLR should be greater than a minimum, $m_{\ell_{\min }}$. Therefore, we need to calculate the mean of the input channel LLRs. In this section we present a theorem for calculation of the input LLR mean to the decoder for DemF.

Theorem 1: For the system described in section II, assuming that the relays use DemF, the channel mean that is input to the iterative decoder for the $i$ th source, $S_{i}$, is given by

$$
\begin{aligned}
& m_{\ell_{i}}^{(\text {DemF })}= \\
& 2 \gamma_{\left(S_{i}, D\right)}+\sum_{j=0}^{r} \epsilon_{\left(S_{i}, R_{j}\right)}\left(1-2 p_{D e m}^{\left(S_{i}, R_{j}\right)}\right) \log \left[\frac{1-p_{D e m}^{\left(S_{i}, R_{j}\right)}}{p_{D e m}^{\left(S_{i}, R_{j}\right)}}\right],
\end{aligned}
$$

where $\gamma_{\left(S_{i}, D\right)}$ is the channel SNR between the $i$ th source and the destination, $p_{D e m}^{\left(S_{i}, R_{j}\right)}$ is the probability of hard decision error at the relay given by equation (8) and $\epsilon_{\left(S_{i}, R_{j}\right)}$ the fraction selected by each relay.

Proof: When the relays use DemF the input LLR to the iterative decoding algorithm at the destination will consist of summation of, $\ell^{\left(S_{i}, D\right)}$ and $\ell^{\left(S_{i}, R_{j}\right)}$ as shown in equation (9) in section II. For the single S-D link the channel LLR mean is calculated as

$$
m_{\ell}^{\left(S_{i}, D\right)}=2 \gamma_{\left(S_{i}, D\right)},
$$

where $\gamma_{\left(S_{i}, D\right)}$ is the channel SNR of the S-D link for the $i$ th source. This is the first term in Theorem 1.

Since we have assumed that the R-D links are perfect, $\ell^{\left(S_{i}, R_{j}\right)}$ represents the LLR of hard decisions at the $j$ th relay. From equation (7) we know that the channel LLR mean for $\ell^{\left(S_{i}, R_{j}\right)}$ depends on $p_{D e m}^{\left(S_{i}, R_{j}\right)}$ the probability that an error occurs when making hard decisions at the $j$ th relay retransmitting $i$ th source signal. If for example we assume $\mathbf{y}^{\left(S_{i}, R_{j}\right)}$ (the results of hard decisions mapped to +1 and -1 instead of 0 and 1$)$ is all-(+1) the channel LLR mean is given by

$$
m_{\ell}^{\left(S_{i}, R_{j}\right)}=\log \left[\frac{1-p_{D e m}^{\left(S_{i}, R_{j}\right)}}{p_{\text {Dem }}^{\left(S_{i}, R_{j}\right)}}\right],
$$

where $p_{D e m}^{\left(S_{i}, R_{j}\right)}$ is calculated according to equation (8).

In general since according to our assumption the all-zero codeword was transmitted by the source, assuming the length of the codeword is $m$, the expected number of $+1 \mathrm{~s}$ in the demodulated sequence is $\left(1-p_{D e m}^{\left(S_{i}, R_{j}\right)}\right) m$ while the expected number of $-1 \mathrm{~s}$ in the sequence is $p_{D e m}^{\left(S_{i}, R_{j}\right)} m$. Therefore, the LLR average is calculated as

$$
\frac{\left[\left(1-p_{D e m}^{\left(S_{i}, R_{j}\right)}\right) m-p_{D e m}^{\left(S_{i}, R_{j}\right)} m\right] \log \left[\frac{1-p_{D e m}^{\left(S_{i}, R_{j}\right)}}{p_{D e m}^{\left(S_{i}, R_{j}\right)}}\right]}{m}=
$$

Since the relay will only forward, $\epsilon_{\left(S_{i}, R_{j}\right)}$ fraction of the demodulated sequence which is equivalent to replacing the unselected positions with zero, the channel LLR mean for the $j$ th relay is given by

$$
m_{\ell}^{\left(S_{i}, R_{j}\right)}=\epsilon_{\left(S_{i}, R_{j}\right)}\left(1-2 p_{\text {Dem }}^{\left(S_{i}, R_{j}\right)}\right) \log \left[\frac{1-p_{D e m}^{\left(S_{i}, R_{j}\right)}}{p_{\text {Dem }}^{\left(S_{i}, R_{j}\right)}}\right] .
$$


Finally, since all the channel LLRs from the sources and relays have a symmetric Gaussian distribution, the mean of their sum is the sum of all the channel LLR means of S-D and R-D links; That is for the $i$ th source $S_{i}$

$$
m_{\ell_{i}}^{(D e m F)}=m_{\ell}^{\left(S_{i}, D\right)}+\sum_{j=0}^{r} m_{\ell}^{\left(S_{i}, R_{j}\right)} .
$$

Corollary. For the system described in section II assuming that the relays use DemF the transmission and the decoding process is successful whenever

$$
m_{\ell_{i}}^{(D e m F)} \geq m_{\ell_{\min }} .
$$

This corollary follows directly from Theorem 1 and the EXIT chart analysis.

\section{Energy Minimization}

In the previous section we discussed the criteria for successful transmission and decoding at the destination for DemF based on input channel LLR means. In this section we present a linear programming model that minimizes the number of transmission bits (i.e. transmission power), subject to the constraint that the decoding is successful. In this section we derive a linear programming model that achieves this task.

In our model the objective is to minimize the number of transmitted bits with the constraint of successful decoding at the destination, where decision variables are $\epsilon_{\left(S_{i}, R_{j}\right)}$, the forwarding fractions of relays.

If DemF is used by the relays, and the $i$ th source has a codeword of length $m_{i}$ to transmit to the destination, the objective function is given by

$$
f(\boldsymbol{\epsilon})=\sum_{i=0}^{s} m_{i}+\sum_{i=0}^{s} \sum_{j=0}^{r} \frac{\epsilon_{\left(S_{i}, R_{j}\right)} m_{i}}{R_{i, j}},
$$

Since in section II we assumed the R-D links are perfect through the use of powerful capacity approaching codes, we can replace $R_{i, j}$ with the capacity of the corresponding channel. We can also omit the constant terms since they have no effect on the optimization. Therefore, the objective function can be simplified to

$$
f(\boldsymbol{\epsilon})=\sum_{i=0}^{s} \sum_{j=0}^{r} \frac{\epsilon_{\left(S_{i}, R_{j}\right)} m_{i}}{C\left(\gamma_{\left(R_{j}, D\right)}\right)},
$$

where $C\left(\gamma_{\left(R_{j}, D\right)}\right)$ is the channel capacity between $j$ th relay and the destination.

To derive the constraints for DemF, we define a variable $g^{\left(S_{i}, R_{j}\right)}$ as

$$
g_{\left(S_{i}, R_{j}\right)}=\left(1-2 p_{\text {Dem }}^{\left(S_{i}, R_{j}\right)}\right) \log \left[\frac{1-p_{D e m}^{\left(S_{i}, R_{j}\right)}}{p_{\text {Dem }}^{\left(S_{i}, R_{j}\right)}}\right],
$$

where the term on the right side is derived in equation (15), and represents the R-D link channel LLR mean before fractional selection at the relays. An $s \times(s r)$ matrix, $G_{S R}$, is defined such that the rows of the matrix represent each source and the columns represent $s r \mathrm{~S}-\mathrm{R}$ channels. The columns are listed in the order of

$$
\left(S_{1}, R_{1}\right) \cdots\left(S_{1}, R_{r}\right)\left(S_{2}, R_{1}\right) \cdots\left(S_{2}, R_{r}\right) \cdots\left(S_{s}, R_{r}\right),
$$

which represents the relays 1 through $r$ forwarding for the first source, and then for the second source, and so on. For the $i$ th, row the only nonzero elements are columns $\left(S_{i}, R_{1}\right)$ to $\left(S_{i}, R_{r}\right)$, where the values are the SNRs of corresponding $\mathrm{S}-\mathrm{R}$ channels. Furthermore, let

$$
\mathbf{g}_{i, S R}=\left[g_{\left(S_{i}, R_{1}\right)}, g_{\left(S_{i}, R_{2}\right)}, \ldots, g_{\left(S_{i}, R_{r}\right)}\right]
$$

represent the row vector of S-R channel SNRs from source $S_{i}$ to all $r$ relays. Matrix $G_{S R}$ is then given by

$$
\left[\begin{array}{cccc}
\mathbf{g}_{1, S R} & \mathbf{0}_{r} & \cdots & \mathbf{0}_{r} \\
\mathbf{0}_{r} & \mathbf{g}_{2, S R} & \cdots & \mathbf{0}_{r} \\
\vdots & \vdots & \ddots & \vdots \\
\mathbf{0}_{r} & \mathbf{0}_{r} & \cdots & \mathbf{g}_{s, S R}
\end{array}\right]
$$

where $\mathbf{0}_{r}$ is a row vector of $r$ zeros. The constraints can be derived using Theorem 1 and matrix $G_{S R}$ as

$$
G_{S R} \boldsymbol{\epsilon} \geq \mathbf{m}_{\ell_{\min }}-2 \gamma_{S D}
$$

where $\epsilon, \mathbf{m}_{\ell_{\text {min }}}$, and $\gamma_{S D}$ are given by equations (11), (12), and (10), respectively. Further constraints are required on $\epsilon$ to obtain a meaningful result, namely that

$$
0 \leq \epsilon_{\left(S_{i}, R_{j}\right)} \leq \epsilon_{\left(S_{i}, R_{j}\right)}^{\prime},
$$

where $\epsilon_{\left(S_{i}, R_{j}\right)}^{\prime} \leq 1$. (Setting $\epsilon_{\left(S_{i}, R_{j}\right)}^{\prime}<1$ implies that there are limits on the resources relay $R_{j}$ is prepared to commit to source $S_{i}$.) Thus, the linear program is described by the linear objective function in (20) and linear constraints in (25)-(26).

\section{EXPERIMENTAL RESULTS}

In this section, through simulation, we show the good practical performance of the estimated solution. To do this we will present two sets of simulations, one to prove that the concept works through a simple example, and another to show the method gives useful and accurate results in a realistic scenario. For all of our simulations we use a $(3,6)$ regular LDPC code to encode the data, and therefore all sources have the same codeword length. Using EXIT chart analysis of the $(3,6)$ regular LDPC code, we have calculated the convergence threshold for channel LLR mean as $m_{\ell_{i}}=2.53$.

\section{A. Simple Example}

For our simple example we consider a system with 2 relays and a source as well as a system with 50 relays and a source. The system is considered simple since we assume that all the links in the system have the same SNR. Therefore, the objective function is a minimum when all the $\epsilon_{\left(S_{i}, R_{j}\right)}$ are the same. For our two relay, single source system, we assume that the normalized SNR on all the links is $-1.5 \mathrm{~dB}$. Using our linear programming model the fraction to be forwarded by the two relays for DemF is $\epsilon^{(\operatorname{DemF})}=0.2926$. Our 50 relay, single source model, is adjusted in such a way to have 


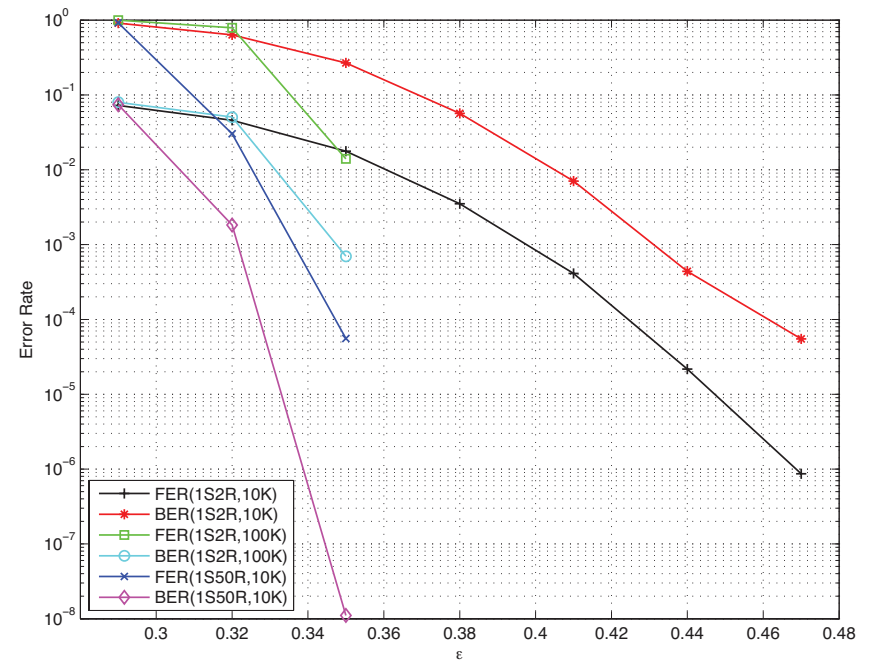

Fig. 2. DemF frame error rate and bit error rate.

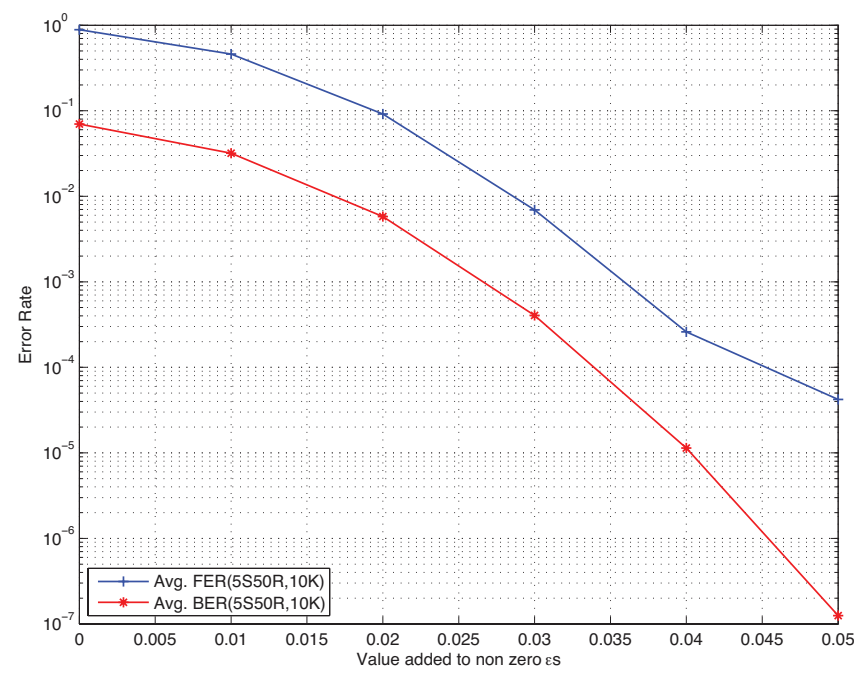

Fig. 3. DemF and multi-source frame error rate and bit error rate.

the same threshold values. Hence, for DemF the normalized SNR on all the links is $-10.32 \mathrm{~dB}$.

Figure 2, shows the resulting BER and FER for DemF with the frame size of $10 \mathrm{k}$ and $100 \mathrm{k}$. Since our linear programming model relies on exit chart analysis it will be more accurate as the frame size or number of relays increase. This effect is captured in these graphs and it can be seen that for the case of 50 relays the curve drops close to the threshold $\epsilon$.

\section{B. Realistic Example}

For our realistic example, we consider a DemF cooperative scheme with 5 sources and 50 relays where the channel SNRs on each link is randomly selected from a Gaussian distribution with mean $-9 \mathrm{~dB}$ and variance $1 \mathrm{~dB}$. Also, we set $\epsilon_{\left(S_{i}, R_{j}\right)}^{\prime}=0.25$ instead of 1 , which takes into account the fact that each node may be unwilling to allocate its entire resources to cooperation. Since the SNRs on each link are different, the problem becomes highly non-trivial. By running our linear programming model we can calculate the values of $\epsilon_{\left(S_{i}, R_{j}\right)}$.

Figure 3 represents the result of simulating such a system. The $x$-axis represents the value that is added to non-zero $\epsilon_{\left(S_{i}, R_{j}\right)}$ (since some relays might not be selected to forward any information for a particular source) that were calculated using the linear programming model. The $y$-axis represents the error rate in terms of BER and FER. We average the BER and FER of the five source to achieve one single curve. As we can see from the graph both curves drop quickly after the threshold value.

\section{REFERENCES}

[1] A. Sendonaris, E. Erkip, and B. Aazhang, "User cooperation diversitypart I: system description," IEEE Trans. Commun., vol. 51, pp. 19271938, Nov. 2003.

[2] A. Sendonaris, E. Erkip, and B. Aazhang, "User cooperation diversity, Part II: Implementation aspects and performance analysis," IEEE Trans. Commun., vol. 51, pp. 1939-1948, Nov. 2003.

[3] A. Nosratinia, T. Hunter, and A. Hedayat, "Cooperative communication in wireless networks," IEEE Commun. Mag., vol. 42, no. 10, pp. 68-73, October 2004.

[4] D. Chen and J. N. Laneman, "Modulation and demodulation for cooperative diversity in wireless systems," IEEE Trans. on Wireless Commun., vol. 5, no. 7, pp. 1785-1794, Jul. 2006.

[5] J. P. K. Chu and R. S. Adve, "Implementation of co-operative diversity using message-passing in wireless sensor networks," in Proc. IEEE Globecom, St. Louis, MO, pp. 1167-1171, Dec. 2005.

[6] A. W. Eckford, J. P. K. Chu, and R. S. Adve, "Low complexity and fractional coded cooperation for wireless networks," IEEE Trans. Wireless Commun., vol. 7, no. 5, pp. 1917-1929, May 2008.

[7] S. ten Brink, "Convergence of iterative decoding," Electron. Lett., vol. 35, no. 10, pp. 806-808, May 1999.

[8] M. Ardakani and F. R. Kschischang, "A more accurate one-dimensional analysis and design of LDPC codes," IEEE Trans. Commun., vol. 52, no. 12, pp. 2106-2114, Dec. 2004.

[9] P. Razaghi and W. Yu, "Bilayer low-density parity-check codes for decode-and-forward in relay channels," IEEE Trans. Inform. Theory, vol. 53, no. 10, pp. 3723-3739, Oct. 2007.

[10] R. Thobaben, "On Distributed Codes with Noisy Relays," in Proc. Asilomar Conference on Signals, Systems, and Computers, Pacific Grove, CA, USA, Oct. 2008.

[11] J. Hu and T. M. Duman, "LDPC codes over ergodic and non-ergodic relay channels," in Proc. 44th Annual Allerton Conference on Communications, Control, and Computing, Monticello, IL, USA, Sep. 2006.

[12] J. P. K. Chu, A. W. Eckford, and R. S. Adve, "Optimization for fractional cooperation in multiple-source multiple-relay systems," in Proc. IEEE International Conference on Communications, Dresden, Germany, Jun. 2009.

[13] T. J. Richardson and R. Urbanke, "The capacity of low-density paritycheck codes under message-passing decoding," IEEE Trans. Inform. Theory, pp. 599-618, Feb. 2001.

[14] D. Divsalar, H. Jin, and R. J. McEliece, "Coding theorems for 'Turbolike' codes," in Proc. 36th Annual Allerton Conference on Communications, Control, and Computing, Monticello, IL, USA, pp. 201-210, Sept. 1998.

[15] T. J. Richardson, M. Shokrollahi, and R. Urbanke, "Design of capacityapproaching irregular low-density parity-check codes," IEEE Trans. Inform. Theory, pp. 619-637, Feb. 2001 